\title{
PRICING OF FINANCIAL DERIVATIVES VIA SIMULATION
}

\author{
Micharl ( $\therefore$ Fu \\ ( ollege of Busiuss and Managinent. \\ Institute for Systems Resiarch \\ University of Marryland at College Park \\ ('ollege Park, Maryland 20742, U.S.A.
}

\begin{abstract}
The word "derivative" has led a ubiquitous existence in the news in recent years. This paper gives a tutorial on financial derivatives and the use of Monte C'arlo simulation techniques for their pricing. We provide the basic financial terminology and key concepts in the field, focusing on options pricing, in particular. Although no prior knowledge of finance is assumed in the exposition, previous experience with stochastic simulations - generation of random inputs and basic statistical output analysis - is requisite.
\end{abstract}

\section{INTRODUCTION}

My major research area is derivative estimation. However, derivative meant in the calculus sense is not what caused Orange County nor Barings Bank to go under. A derivative, simply put and for the purposes of this tutorial, is a financial instrument (or contract) whose value is derived from the values of other instruments, possibly even derivatives themselves or somtthing more basic like the price of a stock, the value of a strock index or the price of a commodity. We will refer to the underlying instrument(s) as the underlying asset(s). Its proper full name is werivative security. Derivatives in the defferental calculus semse are also important in finance as the so-ralled "(irecks," but to avoid confusion, will henceforth be reforred to them as senseturites when nerded.

The pricing of a financial derivative via Monte (arlo simulation can be divided into two basic steps:

1. simulation of the underlying financial asset. $(\mathrm{s})$ and perhaps other non-stationary parameters (e.g., interest rate and stock price volatilit.y)

2. evaluation of the function of those asset (s).

The serond step is merely the definition of the pretticular derivative. It is the first step with which we are most concerned. In practical torms, Mont. Carlo simulation is most likely to be the prefered pricing technique when one or more of the following characteristics is present:

- complicated dynamics characterizing the underlying stochastic processes;

- dependence of the contract on multiple state variables;

- path-dependent contracts.

Aside from the stock dynamics itself, modeling interest rates and stock price volatilities as stochastic processes are two of the most common additional complications. Dependence on multiple state variables generally refers to dependence on a number of different underlying assets. Path dependence considers functions of the entire asset price history versus just the value at a single point in time.

Some key concepts/elements crucial in derivatives pricing include the following:

- differential equations (partial, stochastic);

- no riskless arbitrage;

- risk-neutral measure;

- geometric Brownian motion:

- Black-Scholes pricing model;

- the "(irteks."

We will try to provide a rudimentary understanding of ach of these through examples.

The two most prevalent types of derivative securities are options and futures contracts. In this tutorial, we will consider only option contracts, which can be found as traded securities on the public stock exchanges (c.g., on the Chicago Board Options Exchange (CBOE)) or as privately contracted between two or more parties (. g., a contract hetween an airline and a jot fuel supplier to hedge against fluctuations in fuel prices). In particular, we will with little loss of generality, assume that the underlying asset is a stock. 


\section{SOME BASIC BACKGROUND}

The key components for pricing a stock option contract are the strike price, the expiration datc, the current stock price, the stock volatility, the risk-free interest rate, and the dividends (if any). Without. loss of generality, we will define the "current" time as time 0 . We begin by defining the following variables:

- $S_{t}$ is the stock price at time $t$,

- $S_{0}$ is the initial stock price,

- $r$ is the annualized riskless interest rate (compounded continuously),

- $\mu$ is the drift rate of the stock,

- $\sigma$ is the volatility of the stock,

- $K$ is the strike price of the option contract,

- $T$ is the lifetime (expiration date) of the option contract,

- $J_{t}$ is the net present value of the return of the option at epoch $t$,

- $C$ denotes the net present option value.

A call option on a stock gives the right to buy the stock at a specified price (the strike price) within a specified period of time (the expiration date). A put option on a stock gives the right to sell the stock. A European option can only be exercised at the expiration date $T$, whereas an American option can be exercised at any time up to and including the expiration date. In addition, one can either buy (long) or sell (short) the option itself, creating the following four basic (undiscounted) prositions:

$$
\begin{array}{cl}
\text { long call: } & \left(S_{t}-K^{\prime}\right)^{+} \\
\text {long put: } & \left(K^{\prime}-S_{t}^{\prime}\right)^{+} \\
\text {short call: } & -\left(S_{t}-K^{+}\right)^{+} \\
\text {short put:: } & -\left(K^{*}-S_{t}\right)^{+}
\end{array}
$$

The price of a European call is given by

$$
\begin{aligned}
C & =E\left[\exp \left\{-\int_{0}^{T} r(t) d t\right\}\left(S_{T}-K\right)^{+}\right] \\
& =e^{-r T} E\left[\left(S_{T}^{\prime}-K^{+}\right]\right.
\end{aligned}
$$

where the second equality holds if the interest rate is assumed constant over the horizon. An option which, if it were exercised immediately, would generate a positive cash flow is said to be in the money; otherwise, it is out of the money. Thus, for a (long) call option, an in-the-money option at epoch $t$ is one in which $S_{t}>K^{\prime}$.

The original intent of an options contract was to reduce risk, in contrast to its present notoriety. Consider the following example:
Example: Fuel oil is presently $\$ 1$ per gallon. The fuel oil distributor also offers an option contract to purchase the fuel oil a year from the present at the price of $\$ 1.10$ per gallon, at a cost of $\$ 0.05$ per option for 1 gallon. A company bclieves it will need 1 million gallons of fuel oil in a year, but cannot afford to purchase the fuel at the present time, due to cash flow constraints, for example. It might buy a million options, for a total of $\$ 50,000$, to hedgr against a possible drastic upward turn in the price of fuel oil.

In essence, the option provides a kind of insurance against unexpected drastic fluctuations in the underlying price. If the price had in fact doubled, then the company would have saved itself $\$ 950,000$. If the price went down, on the other hand, the company would just choose not to exercise its option, losing $\$ 50,000$. A futures contract, in contrast, would have committed the company to purchase the fuel at the $\$ 1.10$ price.

Presently, options attracts three types of traders: hedgers - those just described, speculators, and arbitragers. It is the second type which has caused the furor reported in the media. Options give such investors the ability to leverage money, as in the following simple example.

Example: The price of stock ABC is presently $\$ 50$ per share. In addition, there is a corresponding call option available at the strike price of $\$ 50$ and expiration date three months away, at a cost of $\$ 1$ per option. If the stock goes to $\$ 60$ in three months, then the holder of the stock would earn $\$ 10$ per $\$ 50$ invested or a $20 \%$ profit (ignoring transaction costs), whereas the exerciser of the option would earn $\$ 9$ per $\$ 1$ invested or a $900 \%$ profit!

These investors then speculate on future movements of indicators such as stock prices or broader indices, world currency exchange rates, or national interest rates. The third class of traders are arbitragers, traders who look for discrepancies in different markets which allow them to obtain a riskless profit through simultaneous transactions in these markets.

Example: If the exchange rate between the United States and (ireat Britain is $\$ 2$ per pound, and stock $\mathrm{ABC}$ is selling for $\$ 50$ a share in the U.S. and 20 pounds a share in Britain, there is an obvious differcnce of $\$ 10$ a share that an arbitrager could exploit for a riskless profit. This is an example of the concept of riskless arbitrage.

A major assumption that is made in derivatives pricing is the alsence of riskless arbitrage. In essence, this is a statement of market efficiency, for if such an opportunity were to exist, it would be quickly 
exploited and thus subsecuently eliminated through market movements. In practice, there may be minute opportunities that disappear once transaction costs are figured.

There are very few closed-form inalytical results, the most famous one being the Black-Scholes formula to be discussed shorlly. Oftentinu's, approximations based on variations of these model are also proposed. However, for all practical purposes, numerical methods must be employed to do derivatives pricing. There are basically three different approaches taken:

- numerical analysis of the partial differential cquations characterizing the derivative (finite difference methods);

- binomial trees;

- Monte Carlo simulation.

The former two are beyond the scope of this tutorial. Good references include Hull (1993), Stoll and Whaley (1993), Gibson (1991), and Cox and Rubinstein (1985). Only the book by Hull (1993) includes any discussion of Monte Carlo simulation, despite the fact that the technique is widely used in practice.

The ingredients for valuation of a call option based on a single stock via Monte Carlo simulation translate roughly into the following:

1. simulate the stock price dynamics, $S_{t}, 0 \leq t \leq T$;

2. evaluate $\epsilon^{-r \tau}\left(f\left(S_{t}, 0 \leq t \leq \tau\right)-K^{-}\right)^{+}$, where $f$ is some function and $0 \leq \tau \leq T$ represents an exercise time. The interest rate and volatility parameters $r$ and $\sigma$ may also be stochastic processes which need to be simulated. Options based on multiple stocks would simply involve simulating a set of stocks and allowing $f$ to depend on the multiple paths. We discuss some specific examples now.

\section{Examples:}

$$
\begin{aligned}
\epsilon^{-r T}\left(S_{T}-K\right)^{+} & \text {European, } \\
c^{-r \tau}\left(S_{T}-K\right)^{+} & \text {American } \\
\epsilon^{-r T}\left(T^{-1} \int_{0}^{T} S_{l} d l-K\right)^{+} & \text {Asian, } \\
\left.\epsilon_{T-\min }\left\{S_{\ell}, 0 \leq t \leq T\right\}\right)^{+} & \text {lookback. }
\end{aligned}
$$

In order to estimate the value of an European option, one would simply simulate the stock until time $T$, returning $S_{T}-K$ if $S_{T}>K$ and 0 otherwise. This would be repeated a sufficient number of times with a different random number seed for simulating the stock, with confidence intervals formed. The American option problem is more difficult, because there are early axercise option lecisions which must be made. Assuming this cruld be determined, then the procedure is similar to European option valuation.
However, alternative methods have been proposed, as well, that avoid making these decisions explicitly. In the Asian option valuation, one would need to accumulate stock prices at various points along the simulation, and use that to compare to the strike price; thus, this is a path-dependent contract well-suited to Monte Carlo simulation. Otherwise, the procedure is identical to the European option valuation. Similarly, in the lookback option is also path dependent, as the "strike price" in the payoff function here is not the usual a priori specified constant, but rather the minimum of the simulated stock price over the entire horizon.

Thus, what remains is a description of simulating the dynamics of a stock price and other parameters. The celebrated Black-Scholes model prices a European option on a non-dividend-paying stock whose price $S_{t}$ assumed to follow the dynamics given by the stochastic differential equation

$$
d S_{t}=\mu S_{t} d t+\sigma S_{t} d Z_{t}
$$

where $d Z_{t}$ is the standard Wiener process, also known as Brownian motion. This equation also serves to define the usual meaning of drift and volatility. The second term in the equation is called geometric Brownian motion, because $d S_{t} / S_{t}$ follows Brownian motion as opposed to just $S_{t}$ itself (which would not be appropriate, as it would allow negative values for $S_{t}$ unless a reflective boundary were added). Risk-neutral valuation justifies setting $\mu=r$, i.e., in a risk-neutral world, the expected return on all securities must equal the risk-free interest rate. The justification of this is one of the most important results in option pricing (see, e.g., Cox and Ross 1976 or Harrison and Pliska 1981 for a more technical martingale argument).

Monte Carlo simulation of a stock following the dynamics of (1) is done by updating the stock price at $t+\Delta t$ from the price at $t$ in the obvious way via

$$
\Delta S_{t}=r S_{t} \Delta t+\sigma S_{t} Z \sqrt{\Delta t}
$$

where $Z \sim N(0,1)$ is a standard normal random variable, and the appropriate size of $\Delta t$ must be determined. The flexibility of simulation, however, allows one to easily handle other price dynamics, when the choice of geometric Brownian motion does not seem justified. The use of geometric Brownian motion, however, leads to analytical tractability, as the stochastic differential equation (1) can be solved exactly. The solution is found by an application of Ito's Lemma, yielding a lognormally distributed random variable

$$
S_{t}=S_{0} \exp \left[\left(r-\sigma^{2} / 2\right) t+\sigma \sqrt{t} Z\right]
$$


Note that the expected (compounded) return over a finite period is $r-\sigma^{2} / 2$ versus the differential rate $r$ expressed in (1), and that the starting price $S_{0}$ is a scale parameter for the stock price $S_{t}$.

If one were interested in only a single point, such as the terminal price $S_{T}$ in the case of a European option, then only a single random number would have to be generated for every replication estimate. If, however, one were interested in multiple points, say $t_{1}<t_{2}<\ldots<t_{n}=T\left(t_{0}=0\right)$, then the result (3) must be implemented in the correct (Markovian) manner:

Incorrect: Generate $Z_{i} \sim N(0,1)$ i.i.d.

Return $S_{t_{2}}=S_{0} \exp \left[\left(r-\sigma^{2} / 2\right) t_{i}+\sigma \sqrt{t_{i}} Z_{i}\right]$.

Correct: Generate $Z_{i} \sim N(0,1)$ i.i.d.

Return $S_{t_{1}}=S_{0} \exp \left[\left(r-\sigma^{2} / 2\right) t_{i}+\sigma \sum_{j=1}^{i} \sqrt{\Delta t_{j}} Z_{j}\right]$, where $\Delta t_{j}=t_{j}-t_{j-1}$.

Although both methods give the appropriate distribution, only the second method correctly preserves the property of independent increments, as well.

Equations analogous to (1) are often used to model non-stationary stochastic interest rates and volatilities (cf. Hull and White 1987, Johnson and Shanno 1987), e.g.,

$$
\begin{aligned}
d \sigma & =\mu_{\sigma} \sigma d t+\sigma_{\sigma} \sigma d Z_{t}^{(\sigma)} \\
d r & =\mu_{r} r d t+\sigma_{r} r d Z_{t}^{(r)}
\end{aligned}
$$

with the subscripts and superscripts indicating the associated drift, volatility, and Brownian motion components.

In the Black-Scholes model, the interest rate and stock volatility are assumed to remain constant, so the only strochastic process is the stock price itself. Under these assumptions, the following partial differential equation can be derived by using a hedging argument to be described later:

$$
\frac{\partial C}{\partial t}+r S^{\prime} \frac{\partial C^{\prime}}{\partial S^{\prime}}+\frac{1}{2} \sigma^{2} S^{\prime 2} \frac{\partial^{2} C^{\prime}}{\partial S^{\prime 2}}=r C^{\prime},
$$

where $C$ is the value of the option contingent on the stock price $S$. To solve for the European call option, for example, we would apply the boundary condition $C:=\left(S-K^{-}\right)^{+}$at $t=T$, which leads to one of the analytical closed-form Black-Scholes valuation formulas:

$$
C^{\prime}=S_{0} \Phi\left(d_{1}\right)-K e^{-r T} \Phi\left(d_{2}\right),
$$

where

$$
\begin{aligned}
& d_{1}=\frac{\ln \left(S_{0} / K\right)+\left(r+\sigma^{2} / 2\right) T}{\sigma \sqrt{T}}, \\
& d_{2}=\frac{\ln \left(S_{0} / K\right)+\left(r-\sigma^{2} / 2\right) T}{\sigma \sqrt{T}}=d_{1}-\sigma \sqrt{T},
\end{aligned}
$$

and $\Phi(\cdot)$ is the standard normal c.d.f.

\section{SENSITIVITY ESTIMATES}

The concept of hedging risk was touched on earlier. II 'dging positions in options is of crucial importance to financial institutions. By keeping certain positions, a portfolio can he made relatively insensitive to large changes in the parameters affecting the option, such as the asset price and volatility and the interest rate.

Example: The delta of an option (or a derivative sicurity in general) is defined as the sensitivity of the option price with respect to the underlying asset, $\partial C / \partial S$. Assume that the present delta of a call stock option is 0.5 , with the price of the option $\$ 1$ and the underlying stock price $\$ 50$. Then, an investor who has sold 10 option contracts (sold in increments of 100 , for an option to buy 1000 shares total) could hedge by buying $(0.5)(1000)=500$ shares of the stock, so if the stock price goes up by $\$ 1$, the long position would gain $\$ 500$, offsetting (approximately) the loss from the short call option position. Such a position is called a delta neutral position, and the strategy is called delta hedging.

Using this terminology, the Black-Scholes partial differential equation (4) was derived using a deltaneutral position and setting the return on that position equal to the risk-free interest rate, assuming no riskless arbitrage opportunities. Because the delta changes over time, the hedge has to be rebalanced periodically (continuously in the Black-Scholes model).

In any case, it is clear that similar hedging strategies can be pursued with respect to other parameters, as well. A summary of the most commonly used "Cireeks" in hedging are the following:

$$
\begin{aligned}
\Delta & =\frac{\partial C}{\partial S^{\prime}}, \\
\Gamma & =\frac{\partial \Delta}{\partial S}=\frac{\partial^{2} C}{\partial S^{2}}, \\
\Theta & =\frac{\partial C}{\partial t}, \\
\Lambda & =\frac{\partial C}{\partial \sigma}, \\
\rho & =\frac{\partial C}{\partial r} .
\end{aligned}
$$

The implication for simulation is that gradient estimation techniques such as the likelihood ratio method and perturbation analysis (cf. Ho and Cao 1991, Glasserman 1991) can be used here to obtain better and more efficient estimates. Derivation of such algorithms were developed in Broadie and Glasserman (1993) and Fu and $\mathrm{Hu}$ (1995). 


\section{VARIANCE REDUCTION}

Variance reduction techniques are very fruitfully employed in the pricing of financial derivatives. The primary techniques employed are techniques well-known and utilized in stochastic discrete-event simulation:

- common random numbers;

- antithetic variates;

- control variates;

- importance sampling

The use of antithetic variates needs lit.tle further discussion, as it is implemented in the usual straightforward way, by employing pairs of random numbers in the simulation replications. We will discuss the other three techniques in more detail by providing examples of their use.

Common random numbers is sensibly employed in estimating different prices for an option which differ in the settings of the various parameters, e.g., strike price or interest rate, and for use in the usual finite difference estimates of the Greeks of the last section.

Control variates have been very successfully applied for variance reduction. Boyle (1977), in the paper which first introduced Monte Carlo simulation techniques for the pricing of financial derivatives, demonstrated dramatic efficiency improvements for various examples.

Importance sampling can be fruitfully employed for cases where the option is likely to be far out of the money. This is analogous to problems in rare event stochastic simulation, where the rare event here corresponds to the option finishing in the money, since most of the time, the simulation will return 0 .

\section{ASIAN OPTIONS}

Asian options are options on the average asset price. This average could be arithmetic or geometric. The latter affords the luxury of tractability in the case that the underlying stock price follows geometric Brownian motion. Furthermore, in theory, Asian options can be either continurus time or discrete time, but in practice, only discrete time can be realized. The growing popularity of these contracts, especially in over-the-counter exchanges, stem from the risk reduction achieved by averaging over a time period, versus dependence on a single (or few) points in time.

The arithmetic version of Asian options proved difficult to price analytically until the recent work of Geman and Yor (1993), which provided the Laplace transform of the value with respect to the strike price. In contrast, the geometric version is much easier to tackle analytically, because for geometric Brownian motion, the expressions end up involving sums of independent normal random variables. The arithmetic version was recently inverted by Geman and Kydeland (1995) using the fast Fourier transform, and by Fu, Madan, and Wang (1995) using a simple Euler algorithm. Simulation results can also be found there, where the dramatic efficiency improvements (in terms of reduction in the standard error of the estimates) are demonstrated through the use of various control variates, including the geometric average.

Again, the analytical results for this difficult pathdependent security assumed a constant volatility and interest rate, and were done for a single underlying asset. Furthermore, the analysis did not yield the price directly but a Laplace transform, for which the numerical inversion was a non-trivial exercise. It is not too hard to see natural extensions which would create significant obstacles for analytical methods, but would present no substantial additional computational burden on Monte Carlo simulation methods.

\section{AMERICAN-STYLE OPTIONS}

In this section, we illustrate how simulation optimization techniques can be used in derivative pricing by considering an American call option, refuting the claim that "Monte Carlo simulation can only be used for European-style options" (Hull 1993, p.363). Basically, if the pricing problem is viewed as an optimization problem, there are American-style options which can also be priced by using Monte Carlo simulation in conjunction with gradient-based optimization techniques such as those described in Fu (1994ab).

We consider a stock which distributes dividend $D_{j}$ at time $t_{j}, j=1, \ldots, \eta(T)$, where $\eta(T)$ is the number of ex-dividends distributed during the lifetime of the call contract. Following standard models, we assume that after each ex-dividend, the stock price drops by the amount of the ex-dividend, i.e., $S_{t^{+}}=S_{t^{-}}-D_{j}$. For notational convenience, we also denote $t_{0}=0, t_{\eta(T)+1}=T$. We will assume that the ex-dividend amounts $\left\{D_{j}\right\}$ are known (deterministic). Although an American call option can be exercised at any time before the expiration date $T$, under the assumption of a frictionless market, it is well-known that the option should only be exercised, if at all, right before an ex-dividend date or at the expiration date, i.e., only at one of the $t_{j}$ 's. Thus, we can assume that a threshold exercise policy is adopted: there is a stock price $s_{j}\left(\geq K^{*}\right)$ associated with $t_{j}$ such that the option is exercised if (and only if) $S_{t^{-}}>s_{j}$. The European call option can be thought of the special case of $s_{j}=\infty$ for all $j \leq \eta(T)$. 
The sample performance can be written as

$$
\begin{aligned}
& J_{T}=e^{-r T}\left(\sum_{i=1}^{\eta(T)}\left[\prod_{j=1}^{i-1} 1\left\{S_{t_{j}^{-}} \leq s_{j}\right\}\right] 1\left\{S_{t_{i}^{-}}>s_{i}\right\}\right. \\
& \left.\left(S_{t^{-}}-K^{r}\right) e^{r\left(T-t_{1}\right)}+\prod_{j=1}^{\eta(T)} 1\left\{S_{t_{j}^{-}} \leq s_{j}\right\}\left(S_{T}-K^{-}\right)^{+}\right) .
\end{aligned}
$$

The option pricing problem can then be viewed as an optimization problem, whereby the option value is the point at which the expected return $E\left[J_{T}(\theta)\right]$ is maximized with respect to the vector of threshold parameters $\theta=\left[s_{1}, \ldots, s_{\eta(T)}\right]$. To determine the optimal setting of the threshold parameters, we incorporate a gradient estimate $\nabla_{\theta} E\left[J_{T}(\theta)\right]$ into a stochastic approximation algorithm, where the "best guess" of the optimal setting is updated iteratively via

$$
\theta_{n+1}=\Pi_{\odot}\left(\theta_{n}+a_{n} \hat{\nabla} J_{n}\right)
$$

where $\theta_{n}$ is the parameter value at the beginning of iteration $n, \widehat{\nabla} J_{n}=\left[\widehat{\nabla_{1}} J_{n} \cdots \widehat{\nabla}_{p} J_{n}\right]^{T}$ is an estimate of $\nabla J\left(\theta_{n}\right)$ from iteration $n, a_{n}$ is a (positive) sequence of step sizes, and $\Pi_{\Theta}$ is a projection onto $\Theta$. When finite differences are used to estimate $\nabla J\left(\theta_{n}\right),(5)$ is called a Kiefer-Wolfowitz algorithm; when a direct (possibly unbiased) estimator is used for $\nabla J\left(\theta_{n}\right),(5)$ is called a Robbins-Monro-like algorithm. Since the problem is a maximization problem, the stochastic approximation iteration (5) is the positive version of the recursion. The basic underlying assumption in applying stochastic approximation is that the original problem can be solved by finding the zero of the gradient, i.e., by finding $\theta_{*}$, the optimal exercise threshold level, such that $\nabla_{\theta} E\left[J_{T}(\theta)\right]=0$. Of course, in practice, this may lead only to local optimality. The necessary unbiased gradient estimator is derived by using the technique of smoothed perturbation analysis in $\mathrm{Fu}$ and $\mathrm{Hu}$ (1995). Simulation results reported there indicate that the algorithm converges quite quickly, using much less effort than is needed to simply estimate an option payoff to within a penny. In other words, the additional effort needed to estimate an American option using Monte Carlo simulation over what was needed to estimate a European option was negligible.

We note that the procedure described here is tailored to a threshold-based policy, whereas the existence of multiple state variables usually implies that the optimal policy is far more complicated. Such pricing problems can often also be formulated as stochastic dynamic programs, which are then solved by repeated use of Monte Carlo simulation in the backwards recursion. This approach has been pursued by various rescarchers in the finance community, led first by Tilley (1993), but it is clear that using a forwardbased technique like simulation for a backwards algorithm can lead quickly to combinatorial computational complexity. Another simulation-based approach which has some resemblance to the binomial method as well is taken by Broadie and Glasserman (1995), who derive upper and lower bound estimates for the security price that converge asymptotically to the true price.

\section{EXOTIC OPTIONS}

Other types of so-called "exotic" options include the following:

- lookback options - dependence on the maximum or minimum of the stock price during a given interval;

- barrier - dependence on the stock price hitting certain barrier levels during a given interval;

- maximum or minimum of two or more stocks;

- compound options - options on options;

- Bermuda - early exercise allowed only on certain days;

Aside from being exotic, these types of options are also particularly difficult to price using analytical methods, whereas it is clear that aside from the early exercise feature, they are particularly suited to Monte Carlo simulation, because they depend on multiple state variables and/or are path dependent.

\section{CONCLUSIONS}

We have given a brief tutorial on derivatives pricing via Monte Carlo simulation. Through examples, most of the important principles for derivative pricing in general have been illustrated at a very elementary level. In addition, the most fruitful areas for applying Monte Carlo simulation have been outlined. Although the Monte Carlo simulation technique is widely applied in practice, most users are not expert simulationists. Thus, this author feels that there are many opportunities for the simulation community to contribute to making the application to this domain more efficient, particularly in variance reduction techniques, sensitivity analysis, and path-dependent options.

\section{ACKNOWLEDGMENTS}

This work was supported in part by the National Science Foundation under Grant No. NSF EEC 9402384 . 


\section{REFERENCES}

Boyle, P.P. 1977. Options: a Monte Carlo approach. Journal of Financial Economics 4: 323-338.

Broadie, M. and Cilasserman, P. 1993. Estimating security price derivatives using simulation. submitted for publication.

Broadie, M. and (ilasserman, P. 1995. Pricing American-style options. Working paper. Columbia University:

Cox, John ( $\therefore$ and Rubinstein, Mark. 1985. Options markicts. Englewood Clliffs, N.J.: Prentice-Hall.

Cox, J.C. and Ross, S.A. 1976. The valuation of options for alternative stochastic processes," Journal of Financial Economics 3: 145-166.

Fu, M.C. 1994a. Optimization using simulation: a review. Annals of Operations Research 53: 199248.

Fu, M.C. 1994b. A tutorial review of techniques for simulation optimization. In Proceedings of the 1994 Winter Simulation Conference, 149-156.

Fu, M.C. and J.Q. Hu. 1995. Sensitivity analysis for Monte Carlo simulation of option pricing, Probability in the Engineering and Informational Sciences, Vol.9, No.3.

Fu, M.C, Madan, D.B., and T.Wang. 1995. Pricing continuous time Asian options: a comparison of analytical and Monte Carlo methods. presented at the 12th International Conference in Finance, June 1995, Bordeaux, France. also, submitted for publication.

Geman, H. and Kydeland, A. 1995. Domino effect. Risk Volume 8, No. 4.

Geman, H. and Yor, M. 1993. Bessel processes, Asian options and perpetuities. Mathematical Finance 3 , No.4: $34.9-375$.

Gibson, Rajna. 1991. Option valuation: analyzing and pricing standardized optoons contracts. New York: McGraw-Hill.

Gilasserman, P. 1991. Gradient Estimation Via Perturbation Analysis. Kluwer Academic.

Harrison, J.M. and Pliska, S. 1!881. Martingales and stochastic integrals in the theory of continuous trading. Storhastic Processes and their Applications 11: 215-260.

Ho, Y.C. and X.R. ('ao. 1991. Perturbation Analysis and Discrete Event Dynamir Siystoms. Kluwer Academic.

Hull, J. ( $: 1993$. Options, Futures, and Other Derinative Securitıes, 2nd edition. Prentice Hall.

Hull, J.C and White, A. 1987. The pricing of options on assets with stochastic volatilities, Journal of Finance 42: 281-300.
Johnson, H. and Shanno, D. 1987. Option pricing when the variance is changing. Journal of Financial and Quantitative Analysis 22: 143-151.

Stoll, Hans R., and Whaley, Robert E. 1993. Futures and options : theory and applications. Cincinnati, Ohio: South-Western Pub. Co.

'Tilley, J. 1993. Valuing American options in a path simulation model. Transactions of the Society of Actuaries 45: 83-104.

\section{AUTHOR BIOGRAPHY}

MICHAEL C. FU is an Associate Professor of Management Science \& Statistics in the College of Business and Management, with a joint appointment in the Institute for Systems Research, at the University of Maryland at College Park. He received a bachelor's degree in mathematics and bachelor's and master's degrees in electrical engineering from MIT in 1985, and M.S and Ph.D. degrees in applied mathematics from Harvard University in 1986 and 1989, respectively. His research interests include stochastic derivative estimation and simulation optimization, particularly with applications towards manufacturing systems, inventory control, and the pricing of financial derivatives. He teaches courses in applied probability, stochastic processes, discrete-event simulation, and operations management, and in 1995 was awarded the Maryland Business School's annual Allen J. Krowe Award for Teaching Excellence. He is the co-author with Jian-Qiang Hu of the forthcoming book, Conditional Monte Carlo: Gradient Estimation and Optimization Applications, to be published by Kluwer Academic Publishers in 1996. He is a member of IEEE and INFORMS, and on the Program Committee for the Spring 1996 INFORMS National Meeting in Washington, D.C. 